\title{
A Research of Network Congestion Pricing Mechanism in TCP Connection Based on Utility Model
}

\author{
Lingyi $\mathrm{Hu}$ \\ Department of Public Economics, The School of Economics \\ Xiamen University \\ Xiamen, China \\ Lingyihu@163.com
}

\begin{abstract}
Network externality is a vital characteristic of the products in economics of networks. Network congestion will lead to negative network externality when digital products are communicated in the net. It will effectively decrease the negative network externality via developing a suitable pricing mechanism. This paper begins with the utility of a single user and that of the whole network to build a network congestion utility model to analyze it. As TCP connection has an autonomous congestion-detecting mechanism which can increase the utilization of network resources, we regard the TCP connection pricing as the basis of our research.
\end{abstract}

Keywords-network congestion; negative network externality; TCP connection; pricing; utility model

\section{INTRODUCTION}

Congestion is a network condition that has sustained acceleration in which the demands for various network resources including link bandwidth, storage space, the processing capability of processors and so on have been far from the inherent capacity of the net [1]. Simply speaking, each channel has its own specific capacity when information is transmitted in Internet and if the speed that users send bites exceeds a certain value there is likely to appear network congestion. As the number of users of the Internet has increased rapidly these years, the problem of network congestion has been more and more serious. The situation not only reduced the utilization of the net but also leads to overbalance of distributed loads in the net [2]. The resources of network communication have become more scarce which makes solving the problem by pricing feasible.

To solve the problem of network congestion, engineers majoring in computer science, communication and physics have made great efforts in expanding the channel capacity, changing transmission material, improving network facilities and the like. However, it seems not enough to discuss the solution through engineering. Pricing is an appropriate way to solve the problem of distributing scarce resources. Although net providers have adopted flat pricing, users cannot acquire the relevant transmission speed according to his own demand. Users who need high speed prefer to be willing to pay more for it to satisfy his needs while users who do not care about the speed when communicating in the net are more willing to pay less and they can always tolerate the congestion in busy time. As a consequence, the price we can make is different for persons owning different demands. And that is what this paper focuses on

This paper first builds a utility model to explore the feasibility of pricing for network congestion. Then we base the TCP connection as the foundation to discuss how the applications based on TCP connection can be priced. Users depend on TCP connection don't care too much about the transmission speed and they even can accept a low speed but in turn they demand high accuracy. So we can study on how to price for users when TCP connection has accuracy and high-efficiency(means owning the advantage of transmission speed) meanwhile. On the other hand, TCP connection has an autonomous congestion-detecting mechanism which makes it easier to analyze the pricing method comparing with service oriented asyndetic like UDP. This will also lay a foundation for further research.

\section{NEGATIVE NETWORK EXTERNALITY IN NETWORK CONGESTION}

In economics of networks, network externality is the principal characteristic of all kinds of products. Network externality can be divided as positive and negative externality respectively [3]. Negative network externality indicates the utilities of original and new users will decrease as the number of persons who use a certain product is increasing. This phenomenon appears obvious in network congestion. When different users send or receive bites via the Internet, if there exist too much data in one channel which results in message delayed or even abandoned, the sender or the receiver will bear the loss without exception. The messages will need to be given up or totally abandoned. Although the increase in number of users bings about grow in information types and can benefit all users at the same time, network congestion will make their utilities decrease far more than they can acquire in the net.

The result that negative network externality brings is the utility of each user decreases as the number of users of a certain product grows. However, the Internet access pricing adopts flat pricing which means net providers charge fixed fee to a agreed interval like one month, half a year, one year for the usage of network. Through this method of pricing, users can acquire "Best-Effort" service. As a consequence, users who contribute in the network congestion don't have to pay an extra price for their service. And that is that negative network externality contains. 
The method to eliminate this kind of negative network externality is to make every user who continue to use the network to send or receive data in busy time period pay an extra price for the service. And this will in turn counteract the influence the congestion bings. The method can be simplified as the following: When the network congestion occurs, the net providers make a price for each user. If users are urgent to continue to use the net, he or she must pay for the price. Likewise, users who are not willing to pay for the extra price will need to face the situation that they can not continue to use the network properly. To some extent, the method will contribute to the solving of network congestion.

\section{UTILITY MODEL UNDER NETWORK CONGESTION}

At present, the methods regarding to network congestion can be divided as the following three: pricing mechanism based on model, pricing mechanism based on utilization and pice mechanism based on capability [5]. The three methods have solved the problem of network congestion to a certain extent but they do not take the demands of users into consideration so they can not ensure the maximum of utility of the entire network. The utility model in this paper is an economic model based on utility function and aiming at achieving the maximum utility in single person and in the whole net as well.

Scott Shenker indicated in his research that IP Service can be classified as elastic service and inelastic service [6]. Traditional IP Service like WWW, FTP, E-mail are elastic service and Emerging real-time multimedia services like IP telephone and so on are called inelastic service. The latter has strict requirement for available network resources. The two kinds of services are different in utility function so that the ways to analyze are also unlike. In this paper we only take pricing mechanism of elastic service into consideration because it has a strong adaptive capability to network congestion which makes it fittable for our first-step research.

We suppose the largest channel capacity is $Q$ and the speed of information transmission that the $i$ th user has is defined as $r_{i}$. So in the whole IP network, the load of the channel is $\sum_{i=1}^{n} r_{i}$. We define the utilization of network resources is $d=\sum_{i=1}^{n} r_{i} / Q . d$ represents the situation of usage of channel in the net and if the larger $d$ is, the probability that there exists network congestion is higher, and vice versa.

Suppose the benefit the $i$ th user can get from the usage of the net (revenue function) is as followed:

$$
W\left(r_{i}, d\right)=u_{i}\left(r_{i}, d\right)-C_{i}(d) .
$$

In this equation, $u_{i}\left(r_{i}, d\right)$ is the utility the $i$ th user can get through owning the transmission speed of $r_{i}$ and possibly comes across a network congestion at the same time. $C_{i}(d)$ is the cost that the $i$ th user needs to pay for as the utilization of the net is changing [7]. Especially when $d>1$ it means there is a congestion in the net and the user cost represents a congestion cost that package delay and package loss bring about. So (1) has :

$$
\frac{\partial u_{i}}{\partial r_{i}}>0, \frac{\partial^{2} u_{i}}{\partial r_{i}^{2}}<0, \frac{\partial u_{i}}{\partial d}<0 ; \frac{\partial C_{i}}{\partial d}>0, \frac{\partial^{2} C_{i}}{\partial d^{2}}>0
$$

As the purpose of the paper is to analyze the pricing mechanism of network congestion, we only concern about the situation that $d>1$. When $d \leq 1$, the channel is not completely used up and adding to one user will almost has no effect on other users which means the utility functions of users will not change. We use two condition to state the possibility of pricing.

\section{A. Free usage in network congestion}

To make the utility of the $i$ th user maximized, we take the first order condition of equation (1) and get:

$$
\begin{aligned}
& \frac{\partial W\left(r_{i}, d\right)}{\partial r_{i}}=\frac{\partial u_{i}\left(r_{i}, d\right)}{\partial r_{i}}+\frac{\partial u_{i}\left(r_{i}, d\right)}{\partial d} \frac{\partial d}{\partial r_{i}}-\frac{\partial C_{i}(d)}{\partial d} \frac{\partial d}{\partial r_{i}} \\
& =\frac{\partial u_{i}\left(r_{i}, d\right)}{\partial r_{i}}+\frac{1}{Q}\left[\frac{\partial u_{i}\left(r_{i}, d\right)}{\partial d}-\frac{\partial C_{i}(d)}{\partial d}\right]=0 .
\end{aligned}
$$

So we have the result:

$$
\frac{\partial u_{i}\left(r_{i}, d\right)}{\partial r_{i}}=\frac{1}{Q}\left[\frac{\partial C_{i}(d)}{\partial d}-\frac{\partial u_{i}\left(r_{i}, d\right)}{\partial d}\right] .
$$

To make the utility of the whole net maximized, the optimization problem has been transformed to:

$$
\max \sum_{i=1}^{n} W_{i}\left(r_{i}, d\right)=\sum_{i=1}^{n} u_{i}\left(r_{i}, d\right)-\sum_{i=1}^{n} C_{i}(d)
$$

We also take the first order condition of equation (5) and get:

$$
\begin{aligned}
& \frac{\partial u_{i}\left(r_{i}, d\right)}{\partial r_{i}}+\sum_{i=1}^{n} \frac{\partial u_{i}\left(r_{i}, d\right)}{\partial d} \frac{\partial d}{\partial r_{i}}-\sum_{i=1}^{n} \frac{\partial C_{i}(d)}{\partial d} \frac{\partial d}{\partial r_{i}} \\
& =\frac{\partial u_{i}\left(r_{i}, d\right)}{\partial r_{i}}+\frac{1}{Q}\left[\sum_{i=1}^{n} \frac{\partial u_{i}\left(r_{i}, d\right)}{\partial d}-\sum_{i=1}^{n} \frac{\partial C_{i}(d)}{\partial d}\right]=0 .
\end{aligned}
$$

As a consequence, we have a result:

$$
\frac{\partial u_{i}\left(r_{i}, d\right)}{\partial r_{i}}=\frac{1}{Q}\left[\sum_{i=1}^{n}\left(\frac{\partial C_{i}(d)}{\partial d}-\frac{\partial u_{i}\left(r_{i}, d\right)}{\partial d}\right)\right] .
$$

Comparing equation (4) and (7) we can find that when and only when $\frac{\partial C_{i}(d)}{\partial d}=\frac{\partial u_{i}\left(r_{i}, d\right)}{\partial d}$, the two equations are equal and is zero respectively. However, we know from the above that $\frac{\partial C_{i}}{\partial d}>0$ and $\frac{\partial u_{i}}{\partial d}<0$ at the same time which 
indicates the two equations will never be zero. Therefore equation (4) and (7) are not equal. This indicates the maximization of utility of a single user cann't represent the maximization of utility of the whole net and it will bing about loose in utilities of other users. Generally speaking, it is possible to make a corresponding price $p_{e}$ to adjust distribution of the channel to fulfill all users' needs.

\section{B. Price for the network congestion}

$p_{e}$ can be used to measure the marginal congestion cost that will be added to other users when one user is increasing the amount of information he transmits with the speed of $r_{i}$ [8]. So we have:

$$
p_{e}=\frac{1}{Q}\left[\sum_{i=1}^{n}\left(\frac{\partial C_{i}(d)}{\partial d}-\frac{\partial u_{i}\left(r_{i}, d\right)}{\partial d}\right)\right] .
$$

When the number of users in the net is great enough, the influence that change of $r_{i}$ makes on $d$ can be neglectable. So $p_{e}$ can be regarded as a constant that has no relationship with $r_{i}$. Now the revenue function is defined as followed:

$$
W\left(r_{i}, d\right)=u_{i}\left(r_{i}, d\right)-C_{i}(d)-p_{e} r_{i} .
$$

We take the first order fo (9) and get:

$$
\frac{\partial u_{i}\left(r_{i}, d\right)}{\partial r_{i}}-\frac{1}{Q} \frac{\partial C_{i}(d)}{\partial d}=p_{e}
$$

When the number of users in the net is great, the change of every user's congestion cost as $d$ changes tends to be zero. For the $i$ th user:

$$
\frac{\partial u_{i}\left(r_{i}, d\right)}{\partial r_{i}}=p_{e} .
$$

Comparing equation (7) and (9) we find they are equal. This means when the user number is large enough single user's utility maximization equals to that of the whole net which shows all users have achieved maximum utility through the price $p_{e}$. This suggests it is available to control the network congestion via pricing with $p_{e}$.

\section{PRICING MECHANISM OF TCP CONNECTION}

The control of network congestion mainly depends on internal self-adaptive mechanism of TCP protocol. Theoretically speaking, TCP detects the usable bandwidth of network without stops and accordingly adjust the size of the data-sending window and the speed. When there comes the situation of package loss or increase of package delay, TCP will actively narrow the size of the congestion window to release network buffer and to alleviate the network congestion [9]. TCP connection has an autonomous congestion-detecting mechanism. It is stated that TCP protocol that follows AIMD mechanism can ensure the network is working under stable and high-efficiency condition. Moreover, doing the research of pricing mechanism of TCP connection makes for the analysis of pricing methods of connectionless oriented transactions like UDP and the like.

The characteristic of TCP connection is high reliability which means with TCP protocol information will be transmited to the receiver without package loss. If network congestion occurs, the transmission will delay the package send. We conclude several assumptions before studying the pricing mechanism of TCP connection.

1) Each user has his own expected throughput and each network has its max links the net can accommodate. Links here mean a pathway that connects the sender and the receiver.

2) Use the principle of combination of pricing and bidding. If the price one has offered exceeds a certain threshold value the transmission can start, otherwise the request will be rejected.

3) Each user bids for the net resources. It is decided by the network access point that whether the price is up to the threshold value and whether the transmission can start. In the meanwhile whether there are enough links to ensure to complete the message-convey is also under consideration.

Suppose the channel capacity of one net is $Q$ and expected speed of each link is $R$. So the number of links that can exist in the net at the same time is $S=Q / R$.

We assume the utility function of the $i$ th user is $u_{i}\left(s_{i}\right)$ and the function is a strict concave increasing function. The budget of this user, which means the price he is willing to pay for building up the link per unit time is $m_{i}$. To make his utility maximized with this budget, suppose the price of each link is $p$, then when and only when $s_{i} p=m_{i}$ the user's utility function can get to the top point.

According to the analysis of utility model above, when $p$ makes the utility of the whole net maximized, we can say the price is reasonable. So to the entire network the optimization problem has become:

$$
\max \sum_{i=1}^{n} u_{i}\left(s_{i}\right), \text { s.t. } \sum_{i=1}^{n} s_{i} \leq S .
$$

As $s_{i}=m_{i} / p, \quad S=Q / R$, the result is:

$$
\frac{\sum_{i=1}^{n} m_{i}}{p} \leq \frac{Q}{R} .
$$

Namely:

$$
p \geq \frac{R \sum_{i=1}^{n} m_{i}}{Q} .
$$

So when $p$ meets the equation (14), all users will get the maximized utility in this network. The result shows if budget constraints of all users are known, then it is possible to set a certain price $p$ to make the actual request number 
is equal to that of the links the network can afford. At this time the channel has been used sufficiently.

\section{CONCLUSION AND SUGGESTIONS}

Analyzing from the prospective of economics, the Internet is a kind of quasi-public goods with congestion-type or it can be called club goods. It is feasible to reduce the net resources waste caused by network congestion and to solve the problem of low-level usage [10] The paper first builds a utility model by analyzing the maximization of utility of a single user and the whole net. The result shows making proper price for the network congestion makes for the maximization of utilities of all users. Second it discusses the pricing mechanism of TCP connection and get the conclusion that under the condition that all budgets of users are known, a certain price will make utilities of all users maximized.

The analysis and discussion above has some meaning for the preliminary formation of pricing for network congestion. Here lists some other suggestions to solve the problem of network congestion:

1) Continue to apply technology like improving materials of network, broaden the bandwidth and so on. Physical advantages are critical for the speed of network.

2) Developing the congestion pricing mechanism. To make a proper price for busy time according to demands of users is an effective way to maximize utility of the entire net.

3) To perfect network traffic monitoring. Researches of network congestion pricing have gradually transferred to pricing according to network traffic, so to perfect network traffic monitoring is the guarantee of future research.

\section{REFERENCES}

[1] http://baike.baidu.com/view/1452069.htm.

[2] Junfeng He, Yi Zheng, Mingyong Lai, “A Utility Model based on Network Congestion Pricing Strategy,” Journal of Hunan University(Social Sciences), vol 25. No.5, Sept. 2011, pp. 75-80.

[3] Minghong Zhang, Economics of Networks. Beijing: Higher Education Press, 2007.

[4] F. Kelly, Modelsforaself, managed Internet, [C]Philosophical Transactions of the Royal Society, vol. A358, pp.2335-8,2000. http://www.statslab.cam.ac.uk/ frank/smi.html

[5] Junfeng He, Yi Zheng, Mingyong Lai, "A Utility Model based on Network Congestion Pricing Strategy," Journal of Hunan University(Social Sciences), vol 25. No.5, Sept. 2011, pp. 75-80.

[6] Scott Shenker, "Fundamental design issues for the future Internet," (Invited Paper) IEEE journal on selected areas in communications, 1995, 13(7), pp.1176-1188.

[7] Jiaolong Wei, "Network Resource Allocation Based on Game Theory," the Degree of Doctor of Philosophy in Engineering, Huazhong University of Science \& Technology, March, 2004, pp. 71.

[8] Hangtian $\mathrm{Xu}$, "Network Resource Allocation based on Pricing Theory," the Degree of Doctor of Philosophy in Engineering, Beijing University of Posts and Communications, April, 2007, pp. 35.

[9] Xu Fang, "The Study of the Communication Network Resource based on the Pricing, the Degree of Doctor of Philosophy in Engineering, Beijing University of Posts and Communications, June, 2010, pp. 49.

[10] Guocai Wang, "The Study of Flat Pricing and Internet Congestion," Economics Theory Study, vol. 9, 2006, pp. 88-90. 